\section{Canadian normal lung function values}

In this issue of the Canadian Respiratory Journal, Gutierrez et al (pages 414-424) present normal lung function values and prediction equations for white Canadians. They had six laboratories across the country each test approximately 100 volunteer, nonsmoking white adults (the target was 120 each), with appropriate variation in age and sex. Adequate representation of different ages and sex were obtained, although it appeared to be harder to recruit elderly men than elderly women. A full battery of tests was performed on each person. The equipment was not standardized and varied from centre to centre, and some centres performed

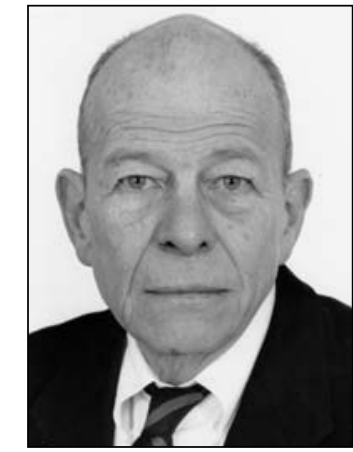

Nick R Anthonisen slightly different tests than others; in other words, the study was done under field conditions. The results were analyzed for each centre and pooled to produce the Canadian model. This was compared with several similar models developed in Europe and the United States (1-5).

Not surprisingly, there were significant centre-to-centre differences in the results, but 'significance' here is a statistical term; none differed greatly from the pooled model, and discrepancies in classification of people as normal or low were not common. It should be noted that such discrepancies in classification are inevitable, especially in 'normal' populations, when the values considered are likely to be close to the normal cut-off values. I am sure that those classified as 'low' were $70 \%$ to $75 \%$ of the predicted normal, as opposed to, say, 40\%. Several features of the pooled models are of interest, at least to me. Total lung capacity declined slightly with age in men but not in women, and functional residual capacity did not change with age. The result in men is at variance with the literature; this could conceivably have been due to the fact that a substantial fraction of the men examined had relatively high body mass index values. If body mass index increased with age, as it usually does, it might have influenced total lung capacity. Instantaneous values of maximum expiratory flow, forced expiratory flow at 50\% of expired vital capacity and forced expiratory flow at $75 \%$ of expired vital capacity had to be log transformed for analysis, and the lower limits of normal were calculated and were $65 \%$ of the mean predicted value, which seems high. Relatively large centre-to-centre differences were observed for these tests and diffusing capacity. These tests are intrinsically hard to do and replicate. Values for airways resistance and its reciprocal, airway conductance, were also quite variable; these tests may not be really interpretable when the results are not extreme.

Generally speaking, the Canadian model agreed well with published normal standards by the Europeans and Americans. This is not surprising given that there is little reason to believe that white Canadians differ greatly from either Europeans or Americans. On the other hand, it is a relief, because, as Gutierrez et al noted, it means that if one is using European or American standards in one's laboratory, they do not need to be changed. However, these results did solve one of my problems. We use Crapo's standards (4) for normal values, and I have been bothered for some time by a

\section{Les valeurs de la fonction pulmonaire normale au Canada}

Dans le présent numéro du Canadian Respiratory Journal, Gutierrez et coll. (pages 414-424) présentent les valeurs de la fonction pulmonaire normale et les équations pronostiques pour les Canadiens de race blanche. Chacun des six laboratoires retenus au pays a évalué environ 100 adultes non fumeurs volontaires (la cible était de 120), affichant une variation convenable selon l'âge et le sexe. Une représentation pertinente des sexes et des âges a été obtenue, même s'il semblait plus difficile de recruter des hommes âgés que des femmes âgés. Chaque individu a subi toute une série de tests. L'équipement n'était pas normalisé et variait d'un centre à l'autre, et certains centres effectuaient des tests légèrement différents que d'autres. Autrement dit, l'étude avait lieu sur le terrain. Les résultats ont été analysés dans chaque centre et regroupés pour produire un modèle canadien. Ce modèle a été comparé à plusieurs modèles similaires mis au point en Europe et aux États-Unis (1-5).

Comme il fallait s'y attendre, on remarquait des différences significatives de résultats entre les centres, mais le terme "significatives » est purement statistique dans ce contexte. Aucun résultat ne différait énormément du modèle groupé, et des écarts de classification entre normaux ou faibles étaient rares. Il faut souligner que ces écarts sont inévitables, surtout dans la population " normale », où les valeurs envisagées sont susceptibles de se rapprocher des valeurs limites normales. Je suis persuadé que ceux qui étaient classés comme « faibles » étaient entre $70 \%$ à $75 \%$ de la normale prévue, par opposition à $40 \%$, disons. Plusieurs caractéristiques du modèle groupé étaient intéressantes, du moins pour moi. La capacité pulmonaire totale diminuait légèrement avec l'âge chez les hommes mais pas chez les femmes, et la capacité résiduelle fonctionnelle ne changeait pas avec l'âge. Les résultats chez les hommes varient de ceux qui figurent dans la documentation scientifique. Ce peut être parce qu'une fraction substantielle des hommes examinés affichaient des valeurs d'indice de masse corporelle élevées. Si l'indice de masse corporelle augmentait avec l'âge, comme c'est généralement le cas, il peut avoir influé sur la capacité pulmonaire totale. Des valeurs instantanées du débit expiratoire maximal, du débit expiratoire forcé à $50 \%$ de la capacité vitale expirée et du débit expiratoire forcé à $75 \%$ de la capacité vitale expirée devaient être transformées en logarithmes pour être analysées, et les limites inférieures de la normale calculée correspondaient à $65 \%$ de la valeur prévue moyenne, ce qui semble élevé. Des différences relativement élevées entre les centres ont été observées à l'égard de ces tests et de la capacité de diffusion. Il est intrinsèque à ces tests d'être difficiles à effectuer et à répliquer. Les valeurs de résistance des voies aériennes et leur réciproque, la conductance aérienne, variaient également beaucoup. Ces tests ne peuvent peut-être pas être vraiment interprétés lorsque les résultats ne sont pas extrêmes.

En général, le modèle canadien correspondait bien aux standards normaux publiés par les Européens et les Américains. Ce n'est pas surprenant puisqu'il y a peu de raisons de croire que les Canadiens de race blanche diffèrent beaucoup des Européens ou des Américains. Par contre, c'est un soulagement, parce que, comme l'ont remarqué Gutierrez et coll., ceux qui utilisent les normes européennes ou américaines dans leur laboratoire n'auront pas à les modifier. Cependant, ces résultats ont réglé l'un de mes problèmes. Nous utilisons les normes de Crapo (4) pour les valeurs normales, et 
high frequency of 'abnormally' low values for lung diffusing capacity to $\mathrm{CO}$ in our laboratory. It turns out that Crapo's predicted normal values are approximately 10\% higher than either the Canadian or European standards, something I had not previously realized. This may reflect the fact that Crapo's subjects were from a relatively high-altitude community (Salt Lake City, Utah) as Gutierrez et al suggest. We probably should change our normal standard.

We can therefore regard the Gutierrez et al study as being largely confirmative of current practices. However, it does point to a potential problem with lung function standards; these results apply to whites, and Canada is becoming less and less white. While there are good predicted normal spirometric values for African-Americans and Mexican-Americans from the United States (6), I am not aware of large systematic studies in Asians, and it seems to me that we need them, although it is very likely that lumping Asians into a single group is not really possible. I suppose it could also be argued that we need normal values for First Nations people, but I suspect that this designation is more sociological than ethnic or genetic, ie, many First Nations people are partly white in origin, and vice versa.

I think the final message from the Gutierrez et al study is that a network of Canadian laboratories was able to do a substantial study with great success, in terms of recruiting, quality control of the data, and production of cogent and coherent results. Further, I am sure that this was done without enormous sums of money; in fact, it may have been done essentially without special funds. Multicentre research networks are now all the rage, for the obvious reason that they can acquire more patients faster than a single centre. There have been successful Canadian research networks, including the Montreal chronic obstructive pulmonary disease network (7) and the national group that examined asthma prevalence (8), and there is a current network looking at chronic obstructive pulmonary disease therapy. More of these would be a good thing, and the Gutierrez et al study reinforces the feasibility of the idea.

Nick R Anthonisen MD Editor-in-Chief, Canadian Respiratory Journal

\section{REFERENCES}

1. Quanjer PH, Trammeling GJ, Cotes JE, Pedersen OF, Peslin R, Yernault JC. Report Working Party Standardization of Lung Function Tests, European Community for Steel and Coal. Official Statement of the European Respiratory Society. Eur Respir J Suppl 1993;16:5-40.

2. Crapo RO, Morris AH, Clayton PD, Nixon CR. Lung volumes in healthy non-smoking adults. Bull Eur Physiopath Respir 1982;18:419-25.

3. Crapo, RO, Morris AH, Gardner RN. Reference spirometric standard values using techniques and equipment that meet ATS recommendations. Am Rev Respir Dis 1981;123:659-64.

4. Crapo RO, Morris AH. Standardized single breath normal values for carbon monoxide diffusing capacity. Am Rev Respir Dis 1981;123:185-9.

5. Miller A, Thornton JC, Warshaw, R, Anderson H, Tierstein AS, Selikoff IJ. Single breath diffusing capacity in a representative sample of the population of Michigan, a large industrial state. Predicted values, lower limits of normal, and frequencies of abnormality by smoking history. Am Rev Respir Dis 1983;127:270-7.

6. Hankinson JL, Odencrantz JR, Fedan KB. Spirometric reference values from a sample of the general U.S. population. Am J Respir Crit Care Med 1999;159:179-87.

7. Collet JP, Shapiro S, Ernst P, Renzi P, Ducruet T, Robinson A, and the PARI-IS Study Steering Committee and Research Group. Effects of an immunostimulating agent on acute exacerbations and hospitalizations in patients with chronic obstructive pulmonary disease. Am J Respir Crit Care Med 1997;156:1719-24.

8. Manfreda J, Becklake MR, Sears MR, et al. Prevalence of asthma symptoms among adults aged 20-44 years in Canada. CMAJ 2001;164:1-7. une fréquence élevée de valeurs « anormalement » faibles de la capacité de diffusion pulmonaire par rapport au débit cardiaque dans notre laboratoire me dérange depuis un certain temps. En fait, les valeurs normales prévues de Crapo sont d'environ $10 \%$ plus élevées que les normes canadiennes ou européennes, ce dont je ne m'étais pas rendu compte auparavant. Ce phénomène s'explique peut-être par le fait que les sujets de Crapo provenaient d'une collectivité où l'altitude est relativement élevée (Salt Lake City, Utah) comme l'ont suggéré Gutierrez et coll. Nous devrions probablement modifier nos standards normaux.

Par conséquent, nous pouvons considérer que l'étude de Gutierrez et coll. confirme en grande partie les pratiques actuelles. Toutefois, elle souligne un problème potentiel des normes de fonction pulmonaire. Ces résultats s'appliquent aux personnes de race blanche, et le Canada est de moins en moins blanc. Bien qu'il existe de bonnes valeurs spirométriques normales prévues pour les AfroAméricains et les Mexico-Américains des États-Unis (6), je ne connais aucune grande étude systématique sur les Asiatiques, et il me semble que nous en avons besoin, même s'il est très probable que le regroupement de tous les Asiatiques dans un même ensemble ne soit pas vraiment possible. Je suppose qu'on pourrait réclamer des valeurs normales pour les autochtones, mais je présume que cette désignation est plus sociologique qu'ethnique ou génétique, c'est-à-dire que de nombreux autochtones sont partiellement blancs, et vice-versa.

Je pense que le message définitif de l'étude de Gutierrez et coll., c'est qu'un réseau de laboratoires canadiens peut procéder à une vaste étude avec grand succès, en matière de recrutement, de contrôle de qualité des données et de production de résultats convaincants et cohérents. De plus, je suis persuadé qu'on y est parvenu sans somme faramineuse, et peut-être même sans fonds spéciaux. Les réseaux de recherche multicentrique font maintenant fureur, pour la raison évidente qu'ils permettent de recruter plus de patients plus rapidement qu'un seul centre. Il existe des réseaux canadiens de recherche qui réussissent, y compris le réseau des maladies pulmonaires obstructives chroniques de Montréal (7) et le groupe national qui examine la prévalence de l'asthme (8), et un réseau est à évaluer le traitement de la maladie pulmonaire obstructive chronique. Il serait bon de compter un plus grand nombre de ces réseaux, et l'étude de Gutierrez et coll. renforce la faisabilité de l'idée.

\section{Nick R Anthonisen MD Rédacteur en chef, Canadian Respiratory Journal}

\section{RÉFÉRENCES}

1. Quanjer PH, Trammeling GJ, Cotes JE, Pedersen OF, Peslin R, Yernault JC. Report Working Party Standardization of Lung Function Tests, European Community for Steel and Coal. Official Statement of the European Respiratory Society. Eur Respir J Suppl 1993;16:5-40.

2. Crapo RO, Morris AH, Clayton PD, Nixon CR. Lung volumes in healthy non-smoking adults. Bull Eur Physiopath Respir 1982;18:419-25.

3. Crapo, RO, Morris AH, Gardner RN. Reference spirometric standard values using techniques and equipment that meet ATS recommendations. Am Rev Respir Dis 1981;123:659-64.

4. Crapo RO, Morris AH. Standardized single breath normal values for carbon monoxide diffusing capacity. Am Rev Respir Dis 1981;123:185-9.

5. Miller A, Thornton JC, Warshaw, R, Anderson H, Tierstein AS, Selikoff IJ. Single breath diffusing capacity in a representative sample of the population of Michigan, a large industrial state. Predicted values, lower limits of normal, and frequencies of abnormality by smoking history. Am Rev Respir Dis 1983;127:270-7.

6. Hankinson JL, Odencrantz JR, Fedan KB. Spirometric reference values from a sample of the general U.S. population. Am J Respir Crit Care Med 1999;159:179-87.

7. Collet JP, Shapiro S, Ernst P, Renzi P, Ducruet T, Robinson A, and the PARI-IS Study Steering Committee and Research Group. Effects of an immunostimulating agent on acute exacerbations and hospitalizations in patients with chronic obstructive pulmonary disease. Am J Respir Crit Care Med 1997;156:1719-24.

8. Manfreda J, Becklake MR, Sears MR, et coll. Prevalence of asthma symptoms among adults aged 20-44 years in Canada. CMAJ 2001;164:1-7. 


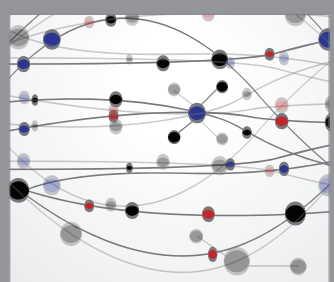

The Scientific World Journal
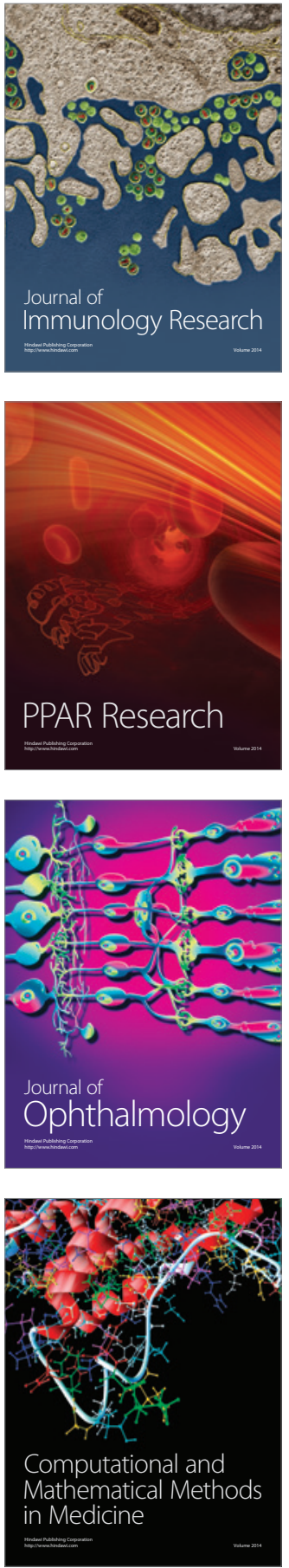

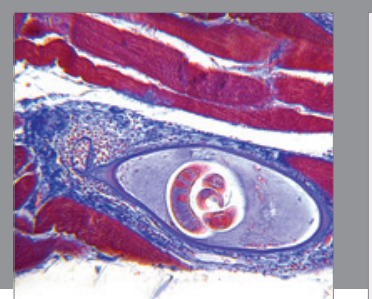

Gastroenterology Research and Practice

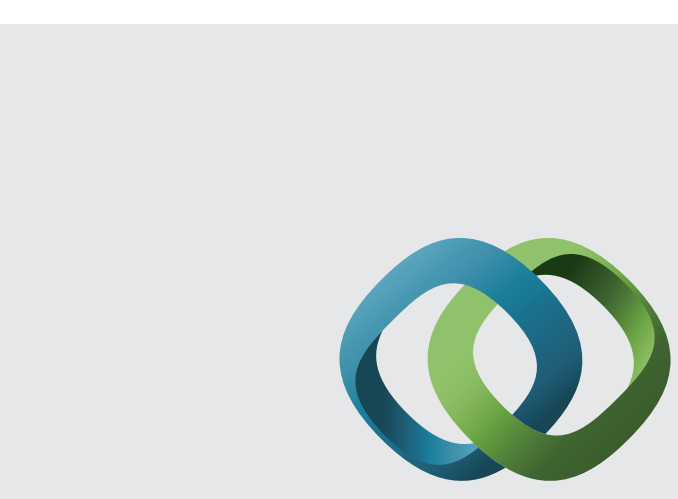

\section{Hindawi}

Submit your manuscripts at

http://www.hindawi.com
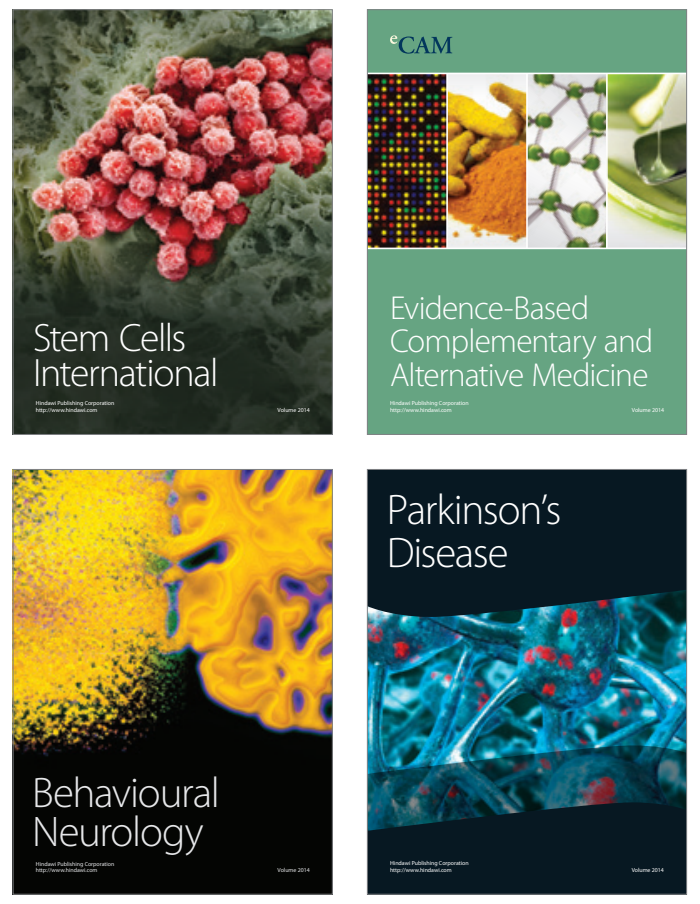
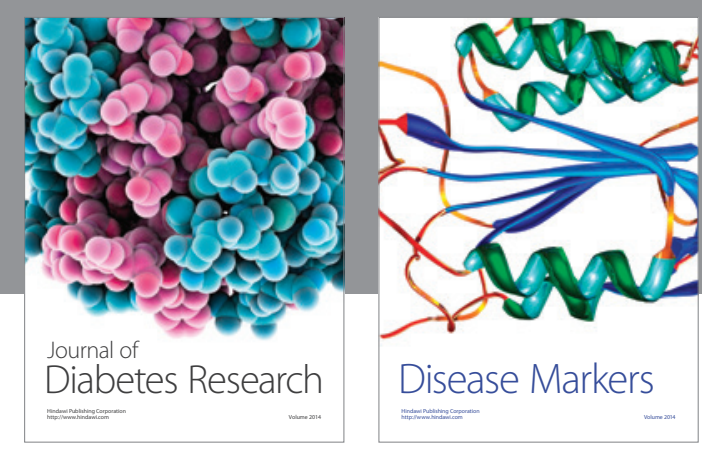

Disease Markers
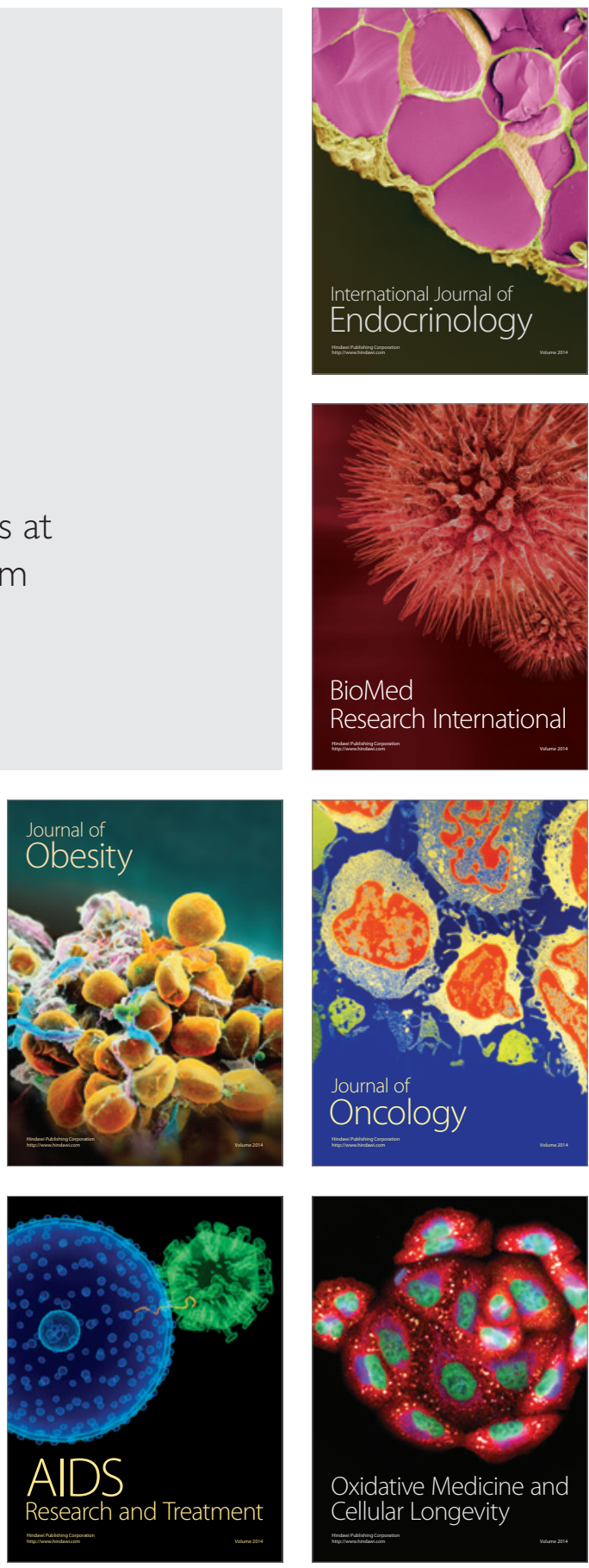